\title{
Dynamic Surface Adaptive Robust Control of Unmanned Marine Vehicles with Disturbance Observer
}

\author{
Pengchao Zhang 1 \\ Key Laboratory of Industrial Automation of Shaanxi Province, Shaanxi University of Technology, Hanzhong, Shaanxi 723000, China \\ Correspondence should be addressed to Pengchao Zhang; snutzpc@126.com
}

Received 30 October 2017; Accepted 18 March 2018; Published 2 May 2018

Academic Editor: Keigo Watanabe

Copyright (C) 2018 Pengchao Zhang. This is an open access article distributed under the Creative Commons Attribution License, which permits unrestricted use, distribution, and reproduction in any medium, provided the original work is properly cited.

This paper presents a dynamic surface adaptive robust control method with disturbance observer for unmanned marine vehicles (UMV). It uses adaptive law to estimate and compensate the disturbance observer error. Dynamic surface is introduced to solve the "differential explosion" caused by the virtual control derivation in traditional backstepping method. The final controlled system is proved to be globally uniformly bounded based on Lyapunov stability theory. Simulation results illustrate the effectiveness of the proposed controller, which can realize the three-dimensional trajectory tracking for UMV with the systematic uncertainty and time-varying disturbances.

\section{Introduction}

Unmanned marine vehicle (UMV) has attracted a number of researchers from all over the world. For the high nonlinearity and the characteristic of being easy to be disturbed by external environment, the control of the UMV is challenged especially for trajectory. With the continuous development of the military and marine economy, UMV needs to complete more complicated tasks accurately. To explore the new nonlinear control strategy for UMV position and trajectory is of great theoretical and practical significance [1-5].

Due to the nonlinear characteristics of the UMV, the backstepping and Lyapunov theory are combined to solve the problem of trajectory tracking control. In [1], considering the Coriolis force and damping force, the backstepping control law is proposed and proved the global exponential stability by the Lyapunov theory. The method of adaptive backstepping is given to design the trajectory tracking controller in view of the slow disturbance from external environment in [2].

Sliding mode control characterized by rapid response, simplicity, and high robustness is used widely for the trajectory control of marine vehicles [3,4]. But the performance is declined by the high-frequency vibration for the switch mode in basic sliding mode. The equipment can be destroyed in the serious case. So, the saturation function and dead zone correction are presented to eliminate the chattering $[5,6]$.
In [7], an adaptive fuzzy backstepping is studied to guarantee the semiglobal congruent eventually boundedness of the close-loop system.

UMV trajectory is inevitably influenced by the external environment in the navigation. The disturbance observer can estimate the external disturbance of the system and observe its characteristics. A nonlinear disturbance observer is adopted for the forward compensation to decline the switch gain of the backstepping controller in [8]. The boundary layer adaptive sliding mode controller based on disturbance observer is also effective in eliminating the chattering for the uncertainty and disturbance in [9]. Aschemann [10] proposed two variable gain feedback nonlinear control methods based on extended linearization technology. Yang et al. [11] give the ship trajectory tracking controller which can resist timevarying environmental disturbance based on disturbance observation, backstepping, and Lyapunov theory. For the "number explosion" in traditional backstepping, Swaroop et al. [12] put forward the dynamic surface control.

This paper presents a composite dynamic surface adaptive robust control method for UMV with disturbance observer to design the position, attitude, and time-varying velocity controllers. The dynamic surface adaptive robust controller is designed for the UMV with disturbance observer. The disturbance observer is for estimating external unknown disturbance and the forward control is for compensation to 


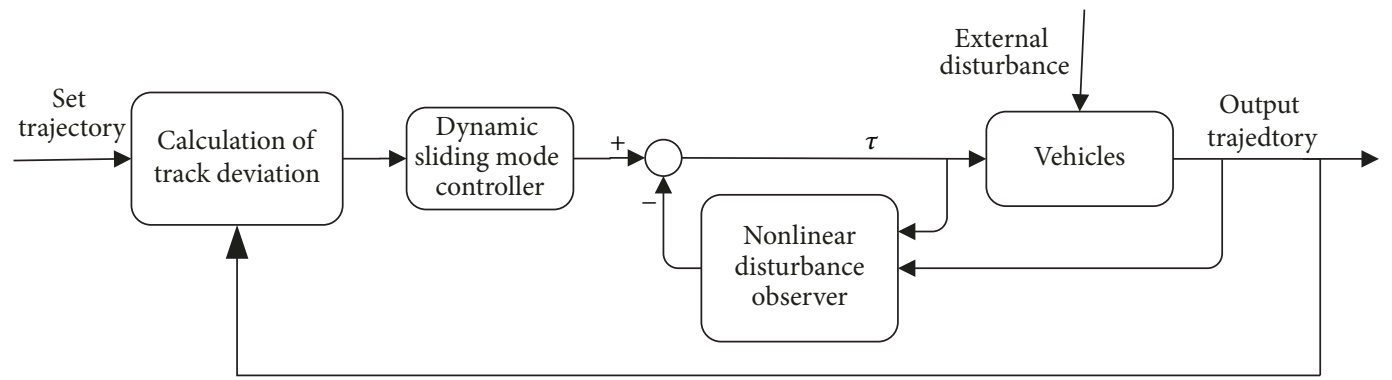

FIgURE 1: Schematic of the control system for UMV.

weaken buffeting. The limit of the disturbance observer error is estimated by the adaptive law. The final controlled system is proved to be globally uniformly bounded based on Lyapunov stability theory.

The remaining parts of this paper are organized as follows. In Section 2, the model of UMV is proposed. In Section 3, the controller design and analysis are shown in detail. At last, the simulation results show the effectiveness of the method in Section 4 and the conclusion is drawn in Section 5.

\section{Model of the UMV}

For a type of UMV, the mathematical model can be expressed as

$$
\begin{aligned}
\dot{\eta} & =J(\psi) \nu \\
M \dot{v} & =\tau+d-C(\nu) \nu-D(\nu) \nu,
\end{aligned}
$$

where $\eta=[x, y, \psi]^{\mathrm{T}}$ is the vector of position $(x, y)$ and yaw angle $\psi$ in the ground coordinate, $v=[u, v, r]^{\mathrm{T}}$ is the vector of advance velocity $u$, drifting velocity $v$, and yaw angular velocity $r$ in hull coordinate. $M$ is the inertial matrix including the added mass. $J(\psi)$ is the transformation matrix and $\mathbf{J}^{-1}(\psi)=\mathbf{J}^{\mathrm{T}}(\psi),\|\mathbf{J}(\psi)\|=1$ :

$$
\mathbf{J}(\psi)=\left[\begin{array}{ccc}
\cos \psi & -\sin \psi & 0 \\
\sin \psi & \cos \psi & 0 \\
0 & 0 & 1
\end{array}\right]
$$

$C(\nu)$ is the matrix of centripetal force and the Coriolis force. $D(\nu)$ is the hydrodynamic resistance and the lifting force moment. $\tau=\left[\tau_{1}, \tau_{2}, \tau_{3}\right]^{\mathrm{T}}$ is the control input vector and $\tau_{1}$ is advance force, $\tau_{2}$ is drifting force, and $\tau_{3}$ is yaw force. $d=\left[d_{1}, d_{2}, d_{3}\right]^{\mathrm{T}}$ is the external disturbance force and $d_{1}$ is lateral disturbance, $d_{2}$ is vertical disturbance, and $d_{3}$ is yaw disturbance.

Assumptions of the UMV are as follows:

(a) The reference trajectory $\boldsymbol{\eta}_{d}$ is smooth, derivable, and bounded.

(b) The disturbance is bounded and $\|\dot{\mathbf{d}}(t)\| \leq C_{d}<\infty$.

\section{Design of the UMV Tracking Controller}

3.1. Design of the Nonlinear Disturbance Observer. The schematic of the UMV tracking control system is shown in
Figure 1. The UMV trajectory is subjected to the environmental disturbances which influence the position and velocity from the expected values. The composite dynamic sliding mode control system can calculate the perfect forces and moment.

Considering the nonlinear kinematic and dynamic equations of the UMV, the nonlinear disturbance observer is designed as

$$
\begin{aligned}
& \widehat{\mathbf{d}}=\boldsymbol{\beta}+\mathrm{K}_{0} \mathbf{M} \boldsymbol{\nu} \\
& \dot{\boldsymbol{\beta}}=-\mathrm{K}_{0} \boldsymbol{\beta}-\mathrm{K}_{0}\left[-\mathrm{C}(\boldsymbol{\nu}) \boldsymbol{\nu}-\mathrm{D} \boldsymbol{\nu}+\tau+\mathrm{K}_{0} \mathrm{M} \boldsymbol{\nu}\right],
\end{aligned}
$$

where $\widehat{\mathbf{d}}=\left[\widehat{d}_{1}, \widehat{d}_{2}, \widehat{d}_{3}\right]^{\mathrm{T}} \in \mathbf{R}^{3}$ is a vector of the disturbance estimate of the observer output and $\mathbf{K}_{0} \in \mathbf{R}^{3 \times 3}$ is a positive definite diagonal matrix, and $\boldsymbol{\beta} \in \mathbf{R}^{3}$ is the intermediate vector for the design.

Define observation error vector $\widetilde{\mathbf{d}}=\left[\widetilde{d}_{1}, \widetilde{d}_{2}, \widetilde{d}_{3}\right]^{\mathrm{T}}$ :

$$
\widetilde{\mathbf{d}}=\mathbf{d}-\widehat{\mathbf{d}} .
$$

Through the derivation of (3) combined with (1), (4), and (5), there is

$$
\begin{aligned}
& \dot{\widehat{\mathbf{d}}}=\dot{\boldsymbol{\beta}}+\mathbf{K}_{0} \mathbf{M} \dot{\boldsymbol{v}}=\mathbf{K}_{0}\left[\mathbf{d}-\left(\boldsymbol{\beta}+\mathbf{K}_{0} \mathbf{M} \boldsymbol{\nu}\right)\right]=\mathbf{K}_{0} \tilde{\mathbf{d}} \\
& \dot{\overrightarrow{\mathbf{d}}}=\dot{\mathbf{d}}-\dot{\widehat{\mathbf{d}}}=\dot{\mathbf{d}}-\mathbf{K}_{0} \tilde{\mathbf{d}} .
\end{aligned}
$$

3.2. Design of Dynamic Surface Adaptive Robust Controller. The control method is based on dynamic surface combined with sliding backstepping. There are two steps in the process.

Step 1. Define the position error vector $\mathbf{z}_{1} \in \mathbf{R}^{3}$ :

$$
\mathbf{z}_{1}=\boldsymbol{\eta}-\boldsymbol{\eta}_{d}
$$

where $\boldsymbol{\eta}_{d}=\left[x_{d}, y_{d}, \psi_{d}\right]^{\mathrm{T}}$ is the vector of the expected position values and yaw angles.

Through the derivation of (8), there is

$$
\dot{\mathbf{z}}_{1}=\mathbf{J}(\psi) \boldsymbol{v}-\dot{\boldsymbol{\eta}}_{d} .
$$

Define virtual vector $\boldsymbol{\alpha}_{1} \in \mathbf{R}^{3}$ and

$$
\boldsymbol{\alpha}_{1}=\mathbf{J}^{-1}(\psi)\left(-\mathbf{K}_{1} \mathbf{z}_{1}+\dot{\boldsymbol{\eta}}_{d}\right),
$$

where $\mathbf{K}_{1} \in \mathbf{R}^{3 \times 3}$ is the positive definite diagonal matrix. 
To avoid the "differential explosion" by virtual control derivation in traditional backstepping control, the first-order filter is introduced according to dynamic surface control. $\boldsymbol{v}_{d} \in$ $\mathbf{R}^{3}$ is the output of the first-order filter of virtual control vector $\boldsymbol{\alpha}_{1}$. That is,

$$
\begin{aligned}
T \dot{\boldsymbol{v}}_{d}+\boldsymbol{\nu}_{d} & =\boldsymbol{\alpha}_{1}, \\
\boldsymbol{v}_{d}(0) & =\boldsymbol{\alpha}_{1}(0),
\end{aligned}
$$

where $T$ is the time constant of the filter.

Step 2. Define the velocity error vector $\mathbf{z}_{2} \in \mathbf{R}^{3}$ as

$$
\mathbf{z}_{2}=\boldsymbol{v}-\boldsymbol{v}_{d} .
$$

According to (1) and (12), there is

$$
\mathbf{M} \dot{\mathbf{z}}_{2}=-\mathbf{C}(\boldsymbol{v}) \boldsymbol{v}-\mathbf{D} \boldsymbol{v}+\boldsymbol{\tau}+\mathbf{d}-\mathbf{M} \dot{\nu}_{d} .
$$

Define the filter error vector $\mathbf{y} \in \mathbf{R}^{3}$ as

$$
\mathbf{y}=\boldsymbol{v}_{d}-\boldsymbol{\alpha}_{1} .
$$

Design the state feedback control law as

$$
\tau=\mathrm{C}(\boldsymbol{v}) \boldsymbol{v}+\mathbf{D} \boldsymbol{v}+\mathbf{M} \dot{\nu}_{d}-\mathbf{K}_{2} \mathbf{z}_{2}-\widehat{\mathbf{d}}-\boldsymbol{\Xi} \widehat{\boldsymbol{\delta}},
$$

where $\mathbf{K}_{2} \in \mathbf{R}^{3 \times 3}$ is the designed positive definite diagonal matrix and $\boldsymbol{\Xi}=\operatorname{diag}\left\{\tanh \left(z_{21} / \varepsilon_{1}\right), \tanh \left(z_{22} / \varepsilon_{2}\right)\right.$, $\left.\tanh \left(z_{23} / \varepsilon_{3}\right)\right\} \in \mathbf{R}^{3} . \varepsilon_{i}$ is the positive constant. $z_{2 i}(i=1,2,3)$ is the weight of $\mathbf{z}_{2} . \widehat{\boldsymbol{\delta}}=\left[\widehat{\delta}_{1}, \widehat{\delta}_{2}, \widehat{\delta}_{3}\right]^{\mathrm{T}} \in \mathbf{R}^{3}$ is the vector of the upper limit of uncertain item $\boldsymbol{\delta}$.

Design the adaptive law of $\widehat{\boldsymbol{\delta}}$ as

$$
\dot{\hat{\boldsymbol{\delta}}}=\Gamma\left[\Xi \mathbf{z}_{2}-\Lambda\left(\widehat{\boldsymbol{\delta}}-\widehat{\boldsymbol{\delta}}^{0}\right)\right],
$$

where $\boldsymbol{\Gamma}=\operatorname{diag}\left\{\gamma_{1}, \gamma_{2}, \gamma_{3}\right\} \in \mathbf{R}^{3 \times 3}$ and $\boldsymbol{\Lambda}=\operatorname{diag}\left\{\sigma_{1}, \sigma_{2}, \sigma_{3}\right\} \in$ $\mathbf{R}^{3 \times 3}$ are the positive definite diagonal matrix. $\widehat{\boldsymbol{\delta}}^{0}=$ $\left[\widehat{\delta}_{1}^{0}, \widehat{\delta}_{2}{ }^{0}, \widehat{\delta}_{3}{ }^{0}\right]^{\mathrm{T}} \in \mathbf{R}^{3}$ is the a priori estimate of $\widehat{\boldsymbol{\delta}}_{i}(i=1,2,3)$.

\subsection{Analysis of Stability. Choose the Lyapunov function as}

$$
V=\frac{1}{2} \mathbf{z}_{1}^{\mathrm{T}} \mathbf{z}_{1}+\frac{1}{2} \mathbf{z}_{2}^{\mathrm{T}} \mathbf{M} \mathbf{z}_{2}+\frac{1}{2} \mathbf{y}^{\mathrm{T}} \mathbf{y}+\frac{1}{2} \widetilde{\boldsymbol{\delta}}^{\mathrm{T}} \boldsymbol{\Gamma}^{-1} \widetilde{\boldsymbol{\delta}}+\frac{1}{2} \widetilde{\mathbf{d}}^{\mathrm{T}} \widetilde{\mathbf{d}},
$$

where $\widetilde{\boldsymbol{\delta}}=\widehat{\boldsymbol{\delta}}-\boldsymbol{\delta}$ is the defined error estimation vector. Through the derivation of (17), there is

$$
\dot{V}=\mathbf{z}_{1}^{\mathrm{T}} \dot{\mathbf{z}}_{1}+\mathbf{z}_{2}^{\mathrm{T}} \mathbf{M} \dot{\mathbf{z}}_{2}+\mathbf{y}^{\mathrm{T}} \dot{\mathbf{y}}+\widetilde{\boldsymbol{\delta}}^{\mathrm{T}} \boldsymbol{\Gamma}^{-1} \dot{\widehat{\boldsymbol{\delta}}}+\widetilde{\mathbf{d}}^{\mathrm{T}} \dot{\tilde{\mathbf{d}}}
$$

For the first item $\mathbf{z}_{11}^{\mathrm{T}} \dot{\mathbf{z}}_{1}$ of (18), considering (9), (10), (12), (13), (14), (15), and $\|\mathbf{J}(\psi)\|=1$, we have

$$
\begin{aligned}
\mathbf{z}_{1}^{\mathrm{T}} \dot{\mathbf{z}}_{1} & =\mathbf{z}_{1}^{\mathrm{T}}\left[\mathbf{J}(\psi)\left(\mathbf{z}_{2}+\mathbf{y}+\boldsymbol{\alpha}_{1}\right)-\dot{\boldsymbol{\eta}}_{d}\right] \\
& =-\mathbf{z}_{1}^{\mathrm{T}} \mathbf{K}_{1} \mathbf{z}_{1}+\mathbf{z}_{1}^{\mathrm{T}} \mathbf{J}(\psi) \mathbf{z}_{2}+\mathbf{z}_{1}^{\mathrm{T}} \mathbf{J}(\psi) \mathbf{y} \\
& \leq-\mathbf{z}_{1}^{\mathrm{T}} \mathbf{K}_{1} \mathbf{z}_{1}+a_{1} \mathbf{z}_{1}^{\mathrm{T}} \mathbf{z}_{1}+\frac{\mathbf{z}_{2}^{\mathrm{T}} \mathbf{z}_{2}}{4 a_{1}}+a_{2} \mathbf{z}_{1}^{\mathrm{T}} \mathbf{z}_{1}+\frac{\mathbf{y}^{\mathrm{T}} \mathbf{y}}{4 a_{2}},
\end{aligned}
$$

where $a_{1}$ and $a_{2}$ are positive constants.
For the second item $\mathbf{z}_{2}^{\mathrm{T}} \mathbf{M} \dot{\mathbf{z}}_{2}$ in (18), considering (13) and (15), there is

$$
\begin{aligned}
& \mathbf{z}_{2}^{\mathrm{T}} \mathbf{M} \dot{\mathbf{z}}_{2}=\mathbf{z}_{2}^{\mathrm{T}}\left[-\mathbf{C}(\boldsymbol{v}) \boldsymbol{v}-\mathbf{D} \boldsymbol{v}+\mathbf{C}(\boldsymbol{v}) \boldsymbol{v}+\mathbf{D} \boldsymbol{v}+\mathbf{M} \dot{\boldsymbol{v}}_{d}\right. \\
& \left.-\mathbf{K}_{2} \mathbf{z}_{2}-\widehat{\mathbf{d}}-\boldsymbol{\Xi} \widehat{\boldsymbol{\delta}}+\mathbf{d}-\mathbf{M} \dot{\boldsymbol{v}}_{d}\right]=-\mathbf{z}_{2}^{\mathrm{T}} \mathbf{K}_{2} \mathbf{z}_{2}-\mathbf{z}_{2}^{\mathrm{T}} \boldsymbol{\Xi} \widehat{\boldsymbol{\delta}} \\
& \quad+\mathbf{z}_{2}^{\mathrm{T}} \widetilde{\mathbf{d}} .
\end{aligned}
$$

For the third item $\mathbf{y}^{\mathrm{T}} \dot{\mathbf{y}}$ in (18), there is

$$
\begin{aligned}
\dot{\mathbf{y}}= & \dot{\mathbf{v}}_{d}-\dot{\boldsymbol{\alpha}}_{1} \\
= & -\frac{\mathbf{y}}{T}+\dot{\mathbf{J}}^{-1}(\psi) \mathbf{K}_{1} \mathbf{z}_{1}+\mathbf{J}^{-1}(\psi) \mathbf{K}_{1} \dot{\mathbf{z}}_{1}-\dot{\mathbf{J}}^{-1}(\psi) \dot{\boldsymbol{\eta}}_{d} \\
& -\mathbf{J}^{-1}(\psi) \ddot{\boldsymbol{\eta}}_{d} .
\end{aligned}
$$

Consider the compact set

$$
\begin{aligned}
& \Omega_{1}=\left\{\left[\mathbf{z}_{1}^{\mathrm{T}}, \mathbf{z}_{2}^{\mathrm{T}}, \mathbf{y}^{\mathrm{T}}\right]^{\mathrm{T}}: V \leq \omega_{0}\right\}, \\
& \Omega_{d} \\
& \quad=\left\{\left[\boldsymbol{\eta}_{d}{ }^{\mathrm{T}}, \dot{\boldsymbol{\eta}}_{d}{ }^{\mathrm{T}}, \ddot{\boldsymbol{\eta}}_{d}{ }^{\mathrm{T}}\right]^{\mathrm{T}}:\left\|\boldsymbol{\eta}_{d}\right\|^{2}+\left\|\dot{\boldsymbol{\eta}}_{d}\right\|^{2}+\left\|\ddot{\boldsymbol{\eta}}_{d}\right\|^{2} \leq B_{0}\right\},
\end{aligned}
$$

where $B_{0}$ and $\varrho_{0}$ are given positive constants. For $\Omega_{1} \times \Omega_{d}$ is also compact set, there are nonnegative continuous functions $\beta(\cdot)$ and the maximum of $\beta(\cdot)$ is $N$.

$$
\begin{aligned}
\left\|\dot{\mathbf{y}}+\frac{\mathbf{y}}{T}\right\| & \leq \beta\left(\mathbf{z}_{1}, \mathbf{z}_{2}, \mathbf{y}, \boldsymbol{\eta}_{d}, \dot{\boldsymbol{\eta}}_{d}, \ddot{\boldsymbol{\eta}}_{d}\right) \\
\mathbf{y}^{\mathrm{T}} \dot{\mathbf{y}} & =-\frac{\mathbf{y}^{\mathrm{T}} \mathbf{y}}{T}+\frac{\mathbf{y}^{\mathrm{T}} \mathbf{y}}{T}+\mathbf{y}^{\mathrm{T}} \dot{\mathbf{y}}=-\frac{\mathbf{y}^{\mathrm{T}} \mathbf{y}}{T}+\mathbf{y}^{\mathrm{T}}\left(\frac{\mathbf{y}}{T}+\dot{\mathbf{y}}\right) \\
& \leq-\frac{\mathbf{y}^{\mathrm{T}} \mathbf{y}}{T}+\alpha_{3} \mathbf{y}^{\mathrm{T}} \mathbf{y}+\frac{N^{2}}{4 \alpha_{3}},
\end{aligned}
$$

where $\alpha_{3}$ is positive constant.

For the fourth item $\widetilde{\boldsymbol{\delta}}^{\mathrm{T}} \boldsymbol{\Gamma}^{-1} \dot{\hat{\boldsymbol{\delta}}}$ of (18) and considering (16), there is

$$
\begin{aligned}
\widetilde{\boldsymbol{\delta}}^{\mathrm{T}} \boldsymbol{\Gamma}^{-1} \dot{\boldsymbol{\delta}} & =\widetilde{\boldsymbol{\delta}}^{\mathrm{T}} \boldsymbol{\Gamma}^{-1} \boldsymbol{\Gamma}\left[\Xi \mathbf{z}_{2}-\boldsymbol{\Lambda}\left(\widehat{\boldsymbol{\delta}}-\widehat{\boldsymbol{\delta}}^{0}\right)\right] \\
& =\mathbf{z}_{2}^{\mathrm{T}} \Xi \widetilde{\boldsymbol{\delta}}-(\widehat{\boldsymbol{\delta}}-\boldsymbol{\delta})^{\mathrm{T}} \boldsymbol{\Lambda}\left(\widehat{\boldsymbol{\delta}}-\widehat{\boldsymbol{\delta}}^{0}\right) \\
& \leq \mathbf{z}_{2}^{\mathrm{T}} \Xi \widetilde{\boldsymbol{\delta}}-\frac{1}{2} \widetilde{\boldsymbol{\delta}}^{\mathrm{T}} \boldsymbol{\Lambda} \widetilde{\boldsymbol{\delta}}+\frac{1}{2}\left(\boldsymbol{\delta}-\widehat{\boldsymbol{\delta}}^{0}\right)^{\mathrm{T}} \boldsymbol{\Lambda}\left(\boldsymbol{\delta}-\widehat{\boldsymbol{\delta}}^{0}\right) .
\end{aligned}
$$

For the fifth item $\widetilde{\mathbf{d}}^{\mathrm{T}} \dot{\tilde{\mathbf{d}}}$ of (18), considering (7), there is

$$
\begin{aligned}
\tilde{\mathbf{d}}^{\mathrm{T}} \dot{\tilde{\mathbf{d}}} & =\widetilde{\mathbf{d}}^{\mathrm{T}}\left(\dot{\mathbf{d}}-\mathbf{K}_{0} \widetilde{\mathbf{d}}\right)=\tilde{\mathbf{d}}^{\mathrm{T}} \dot{\mathbf{d}}-\widetilde{\mathbf{d}}^{\mathrm{T}} \mathbf{K}_{0} \widetilde{\mathbf{d}} \\
& \leq a_{4} \widetilde{\mathbf{d}}^{\mathrm{T}} \widetilde{\mathbf{d}}+\frac{C_{d}{ }^{2}}{4 a_{4}}-\widetilde{\mathbf{d}}^{\mathrm{T}} \mathbf{K}_{0} \widetilde{\mathbf{d}},
\end{aligned}
$$

where $a_{4}$ is positive constant. 
According to (19), (20), (23), (24), and (25), there is

$$
\begin{aligned}
\dot{V} \leq & -\mathbf{z}_{1}^{\mathrm{T}} \mathbf{K}_{1} \mathbf{z}_{1}+a_{1} \mathbf{z}_{1}^{\mathrm{T}} \mathbf{z}_{1}+\frac{\mathbf{z}_{2}^{\mathrm{T}} \mathbf{z}_{2}}{4 a_{1}}+a_{2} \mathbf{z}_{1}^{\mathrm{T}} \mathbf{z}_{1}+\frac{\mathbf{y}^{\mathrm{T}} \mathbf{y}}{4 a_{2}} \\
& -\mathbf{z}_{2}^{\mathrm{T}} \mathbf{K}_{2} \mathbf{z}_{2}+\mathbf{z}_{2}^{\mathrm{T}} \widetilde{\mathbf{d}}-\frac{\mathbf{y}^{\mathrm{T}} \mathbf{y}}{T}+a_{3} \mathbf{y}^{\mathrm{T}} \mathbf{y}+\frac{N^{2}}{4 a_{3}}-\mathbf{z}_{2}^{\mathrm{T}} \boldsymbol{\Xi} \boldsymbol{\delta} \\
& -\frac{1}{2} \widetilde{\boldsymbol{\delta}}^{\mathrm{T}} \boldsymbol{\Lambda} \widetilde{\boldsymbol{\delta}}+\frac{1}{2}\left(\boldsymbol{\delta}-\widehat{\boldsymbol{\delta}}^{0}\right)^{\mathrm{T}} \boldsymbol{\Lambda}\left(\boldsymbol{\delta}-\widehat{\boldsymbol{\delta}}^{0}\right)+a_{4} \widetilde{\mathbf{d}}^{\mathrm{T}} \widetilde{\mathbf{d}} \\
& +\frac{C_{d}{ }^{2}}{4 a_{4}}-\widetilde{\mathbf{d}}^{\mathrm{T}} \mathbf{K}_{0} \tilde{\mathbf{d}} .
\end{aligned}
$$

Considering $0 \leq|a|-a \tanh (a / \varepsilon) \leq 0.2785 \varepsilon$ (for $\varepsilon>0$, $a \in R)$ and $z_{2 i} \widetilde{d}_{i} \leq\left|z_{2 i}\right| \cdot\left|\widetilde{d}_{i}\right| \leq\left|z_{2 i}\right| \delta_{i}$, we can have

$$
\begin{aligned}
\dot{V} \leq & -\left[\lambda_{\min }\left(\mathbf{K}_{1}\right)-a_{1}-a_{2}\right] \mathbf{z}_{1}^{\mathrm{T}} \mathbf{z}_{1} \\
& -\left[\lambda_{\min }\left(\mathbf{K}_{2}\right)-\frac{1}{4 a_{1}}\right] \mathbf{z}_{2}^{\mathrm{T}} \mathbf{z}_{2} \\
& -\left[\frac{1}{T}-\frac{1}{4 a_{2}}-a_{3}\right] \mathbf{y}^{\mathrm{T}} \mathbf{y}-\left[\lambda_{\min }\left(\mathbf{K}_{0}\right)-a_{4}\right] \widetilde{\mathbf{d}}^{\mathrm{T}} \widetilde{\mathbf{d}} \\
& -\frac{1}{2} \lambda_{\min }(\boldsymbol{\Lambda} \boldsymbol{\Gamma}) \frac{1}{2} \widetilde{\boldsymbol{\delta}}^{\mathrm{T}} \boldsymbol{\Gamma}^{-1} \widetilde{\boldsymbol{\delta}}+0.2785 \mathbf{E}^{\mathrm{T}} \boldsymbol{\delta}+\frac{N^{2}}{4 a_{3}} \\
& +\frac{1}{2}\left(\boldsymbol{\delta}-\widehat{\boldsymbol{\delta}}^{0}\right)^{\mathrm{T}} \boldsymbol{\Lambda}\left(\boldsymbol{\delta}-\widehat{\boldsymbol{\delta}}^{0}\right)+\frac{C_{d}{ }^{2}}{4 a_{4}} \leq-\mu V+C,
\end{aligned}
$$

where

$$
\begin{aligned}
& \mathbf{E}=\left[\varepsilon_{1}, \varepsilon_{2}, \varepsilon_{3}\right]^{\mathrm{T}} \\
& \mu=\min \left\{2\left[\lambda_{\min }\left(\mathbf{K}_{1}\right)-a_{1}-a_{2}\right], 2\left[\frac{1}{T}-\frac{1}{4 a_{2}}-a_{3}\right],\right. \\
& \frac{2\left[\lambda_{\min }\left(\mathbf{K}_{2}\right)-1 / 4 a_{1}\right]}{\lambda_{\max }(\mathbf{M})}, \lambda_{\min }(\mathbf{\Lambda} \boldsymbol{\Gamma}) \text {, } \\
& \left.2\left[\lambda_{\min }\left(\mathbf{K}_{0}\right)-a_{4}\right]\right\} \\
& C=0.2785 \mathbf{E}^{\mathrm{T}} \boldsymbol{\delta}+\frac{N^{2}}{4 a_{3}}+\frac{1}{2}\left(\boldsymbol{\delta}-\widehat{\boldsymbol{\delta}}^{0}\right)^{\mathrm{T}} \boldsymbol{\Lambda}\left(\boldsymbol{\delta}-\widehat{\boldsymbol{\delta}}^{0}\right) \\
& +\frac{C_{d}^{2}}{4 a_{4}} \\
& \lambda_{\text {min }}\left(\mathbf{K}_{1}\right)-a_{1}-a_{2}>0 \\
& \lambda_{\text {min }}\left(\mathbf{K}_{2}\right)-\frac{1}{4 a_{1}}>0 \\
& \frac{1}{T}-\frac{1}{4 a_{2}}-a_{3}>0 \\
& \lambda_{\min }(\Lambda \Gamma)>0 \\
& \lambda_{\text {min }}\left(\mathbf{K}_{0}\right)-a_{4}>0 \text {, }
\end{aligned}
$$

where $\lambda_{\min }(\cdot)$ is the minimal eigenvalue of the matrix and $\lambda_{\text {max }}(\cdot)$ is the maximal eigenvalue of the matrix.

According to the above analysis, the theorem is drawn as follows.

Theorem 1. Considering the kinematic dynamic model of the trajectory tracking ship with three degrees of freedom satisfies the assumptions, there are adaptive disturbance observers as (3) and (4), first-order filter as (11), and control laws as (15) and (16) which can guarantee the global uniform ultimate boundedness of the tracking system. Choose the proper $\mathbf{K}_{0}, \mathbf{K}_{1}$, $\mathbf{K}_{2}, \Lambda, \Gamma, \varepsilon_{i}, \widehat{\boldsymbol{\delta}}_{i}^{0}$, and T to satisfy (31)-(35). The UMV can be tracked with high precision.

Proof. To solve (27), there is

$$
0 \leq V(t) \leq \frac{C}{\mu}+\left[V(0)-\frac{C}{\mu}\right] e^{-\mu t} .
$$

So, $V(t)$ is uniform ultimate boundedness. Combining (17) and (36), there is

$$
0 \leq\left\|\mathbf{z}_{1}\right\| \leq \sqrt{\frac{2 C}{\mu}+2\left[V(0)-\frac{C}{\mu}\right] e^{-\mu t}} .
$$

For arbitrary $\mu_{\mathbf{z}_{1}} \geq \sqrt{2 C / \mu}$ and existing constant $T_{\mathbf{z}_{1}}$, there is $\left\|\mathbf{z}_{1}\right\| \leq \mu_{\mathbf{z}_{1}}$ for all $t>T_{\mathbf{z}_{1}}$. That is, the position error vector $\mathbf{z}_{1}$ is convergent to the compact set $\Omega_{\mathbf{z}_{1}}=\left\{\mathbf{z}_{1} \in R^{3}\right.$ | $\left.\left\|\mathbf{z}_{1}\right\| \leq \mu_{\mathbf{z}_{1}}\right\}$. The proper adjustment of $\mathbf{K}_{0}, \mathbf{K}_{1}, \mathbf{K}_{2}, \boldsymbol{\Lambda}, \boldsymbol{\Gamma}, \varepsilon_{i}, \widehat{\boldsymbol{\delta}}_{i}^{0}$, and $T$ can make $\Omega_{\mathbf{z}_{1}}$ arbitrarily small. That is, we can get the high precision tacking.

\section{Simulation Example}

In this paper, a supply vessel is taken as the simulation example in [13]. The length of the vessel is $76.2 \mathrm{~m}$ and the mass is $4.591 \times 10^{6} \mathrm{~kg}$. The parameter matrixes are as follows:

$$
\begin{gathered}
\mathbf{M}=10^{6} \times\left[\begin{array}{ccc}
5.3122 & 0 & 0 \\
0 & 8.2831 & 0 \\
0 & 0 & 3745.4
\end{array}\right] \\
\mathbf{C}(\boldsymbol{v})=10^{6} \times\left[\begin{array}{ccc}
0 & 0 & 8.2831 v \\
0 & 0 & 5.3122 u \\
8.2831 v & -5.3122 u & 0
\end{array}\right] \\
\mathbf{D}=10^{4} \times\left[\begin{array}{ccc}
5.0242 & 0 & 0 \\
0 & 27.299 & -439.33 \\
0 & -439.33 & 41894
\end{array}\right] .
\end{gathered}
$$

The disturbance from external environment is described by a first-order Gauss-Markov process as

$$
\begin{aligned}
& \mathbf{d}=\mathbf{J}^{\mathrm{T}}(\psi) \mathbf{b} \\
& \dot{\mathbf{b}}=-\mathbf{T}_{c} \mathbf{b}+\boldsymbol{\rho} \mathbf{n},
\end{aligned}
$$


where $\mathbf{b} \in \mathbf{R}^{3}$ is the external disturbance in the earth reference coordinate.

$\mathbf{T}_{c}=\operatorname{diag}\left\{10^{3}, 10^{3}, 10^{3}\right\}$ is the designed time constant diagonal matrix. $\mathbf{n} \in \mathbf{R}^{3}$ is the zero mean Gaussian white noise vector. $\boldsymbol{\rho}=10^{4} \times \operatorname{diag}\{5,5,50\}$ is amplitude matrix.

Assume that the expected trajectory is

$$
\begin{aligned}
& x_{d}=500 \sin (0.02 t), \\
& y_{d}=500[1-\cos (0.02 t)], \\
& \psi_{d}=0.01 t .
\end{aligned}
$$

The initial position and velocity vector are

$$
\begin{aligned}
& {[x(0), y(0), \psi(0), u(0), v(0), r(0)]^{\mathrm{T}}} \\
& \quad=\left[100 \mathrm{~m}, 200 \mathrm{~m}, \frac{\pi}{4}, 0 \mathrm{~m} / \mathrm{s}, 0 \mathrm{~m} / \mathrm{s}, 0 \mathrm{rad} / \mathrm{s}\right]^{\mathrm{T}} .
\end{aligned}
$$

The initial state of observer is $\mathbf{b}(0)=[0,0,0]^{\mathrm{T}}$ and control parameters matrixes, respectively, are

$$
\begin{aligned}
& K_{0}=\operatorname{diag}\{2,2,2\}, \\
& \mathbf{K}_{1}=0.08 \times \operatorname{diag}\{1,1,1\}, \\
& \mathbf{K}_{2}=10^{6} \times \operatorname{diag}\left\{1,1,10^{3}\right\}, \\
& \Lambda=10^{-8} \times \operatorname{diag}\left\{1,1,10^{-3}\right\}, \\
& \Gamma=10^{6} \times \operatorname{diag}\left\{1,1,10^{3}\right\}, \\
& \varepsilon_{1}=\varepsilon_{2}=0.005, \\
& \varepsilon_{3}=0.0001, \\
& \widehat{\delta}_{1}^{0}=\widehat{\delta}_{2}^{0}=\widehat{\delta}_{3}^{0}=0.1 .
\end{aligned}
$$

The filter time constant is $T=0.3$. So, in the conditions of $a_{1}>2.5 \times 10^{-7}, 0<a_{2}<0.2-\alpha_{1}, 0<a_{3}<10 / 3-1 /\left(4 a_{2}\right)$, and $0<a_{4}<2$, (37) $-(39)$ are satisfied.

The simulation results are shown in Figures 2-4.

Figure 2 shows the expected and actual position and yaw angle curves. From the results we can see that the UMV can reach the expected trajectory at 15 seconds despite the external disturbance. Figure 3 shows the external disturbance d and its estimation $\widehat{\mathbf{d}}$. So, the disturbance observer can estimate the external unknown disturbance. From Figures 3 and 4 we can see that the estimation $\widehat{\mathbf{d}}$ can reach actual $\mathbf{d}$ using the nonlinear disturbance observer and the controller switch gain $\widehat{\boldsymbol{\delta}}$ can be very small. This can weaken the drifting of the controller. So, the controller has the high robust and good performance.

\section{Conclusions}

During the actual ocean voyage, the UMV suffers external environmental disturbance. This paper assumes that external
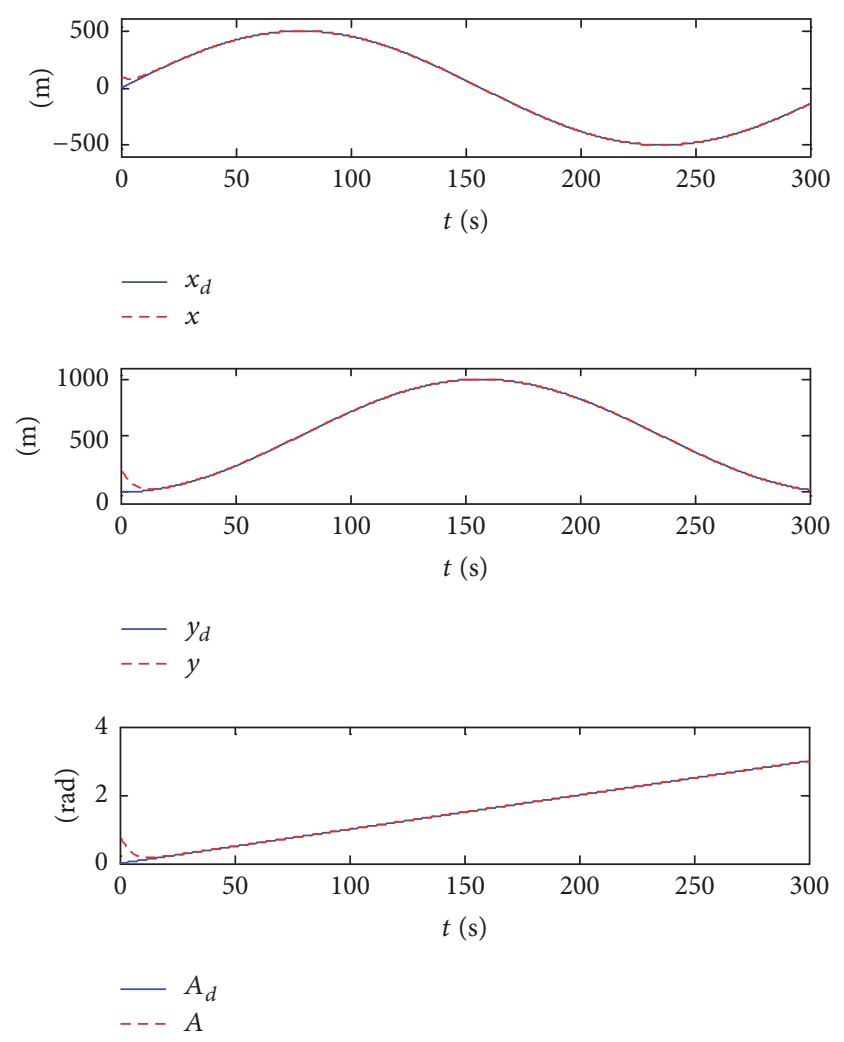

FIGURE 2: Curves of expected trajectory $\boldsymbol{\eta}_{d}$ and actual trajectory $\boldsymbol{\eta}$.
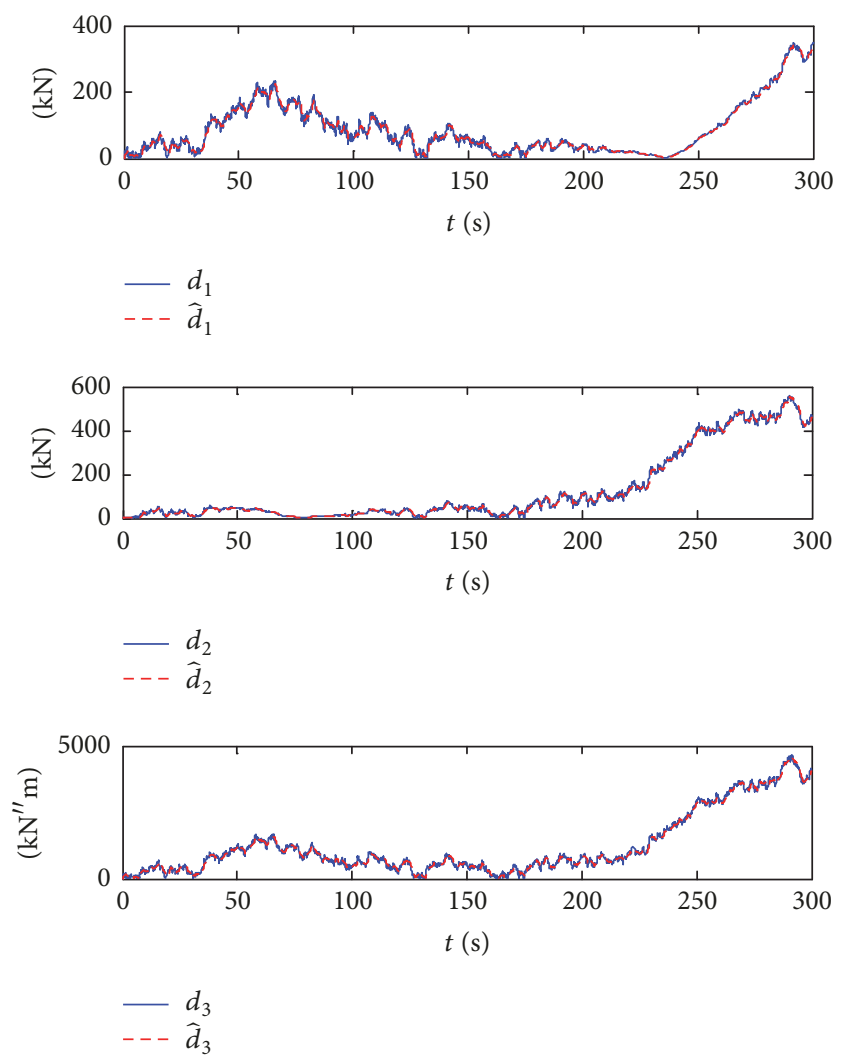

FIgURE 3: Curves of external environment disturbances $\mathbf{d}$ and estimations $\widehat{\mathbf{d}}$. 

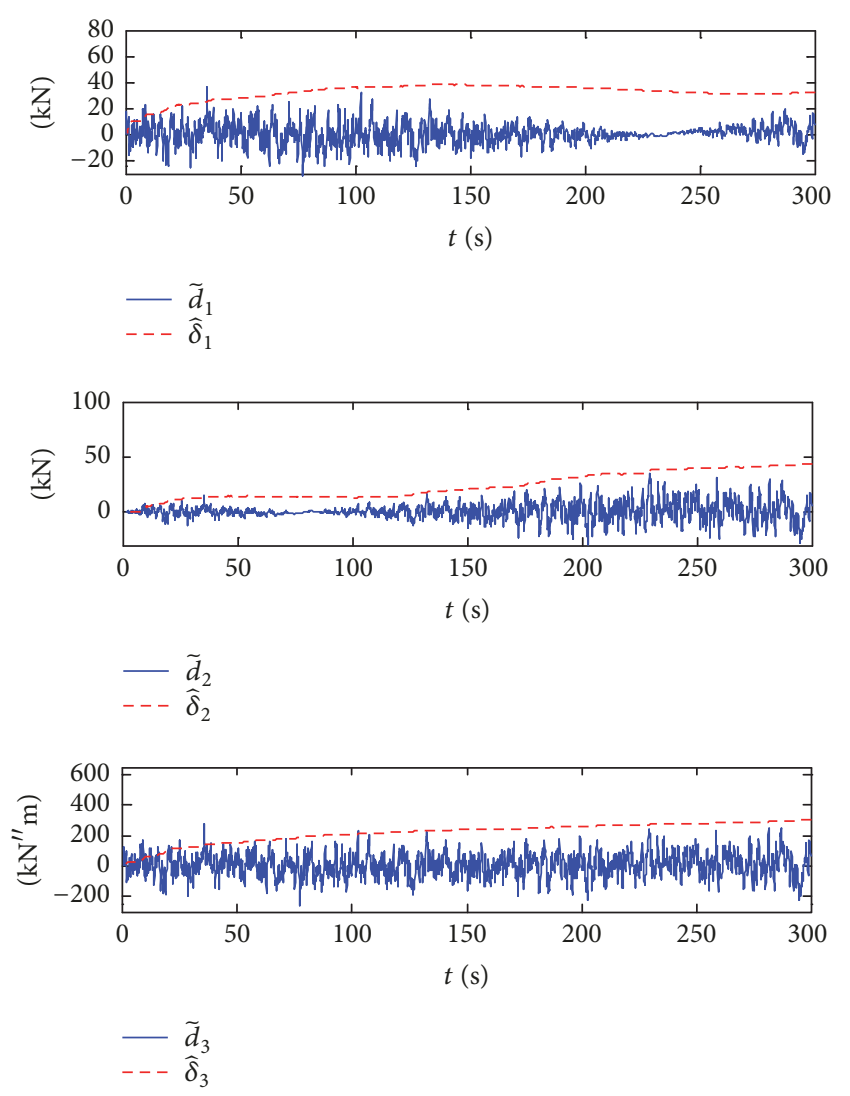

FIgURE 4: Curves of disturbances observer errors $\widetilde{\mathbf{d}}$ and their upper bound estimations $\widehat{\boldsymbol{\delta}}$.

disturbance and its boundedness are unknown. Combined with nonlinear disturbance observer, dynamic surface control, and adaptive robust backstepping, the dynamic surface adaptive robust controller is designed for the UMV with disturbance observer. The disturbance observer is for estimation of external unknown disturbance and the forward control is for compensation to weaken buffeting. The limit of the disturbance observer error is estimated by the adaptive law. The dynamic surface control is introduced to solve the "differential explosion." At last, the simulation of an UMV shows the high precision of trajectory tacking. This is meaningful in engineering practice.

\section{Conflicts of Interest}

The author declares that there are no conflicts of interest regarding the publication of this paper.

\section{Acknowledgments}

This work was supported by Key Project of Shaanxi Provincial Education Department under Grant no. 16JS017 and Shaanxi Provincial Science and Technology Department Industrial Research Project under Grant no. 2016GY-070.

\section{References}

[1] A. P. Mouritz, E. Gellert, P. Burchill, and K. Challis, "Review of advanced composite structures for naval ships and submarines," Composite Structures, vol. 53, no. 1, pp. 21-41, 2001.

[2] J. M. Godhavn, T. I. Fossen, and S. P. Berge, "Non-linear and adaptive backstepping designs for tracking control of ships," International Journal of Adaptive Control and Signal Processing, vol. 12, no. 8, pp. 649-670, 1998.

[3] X. Yang, T. Wang, J. Liang, G. Yao, and M. Liu, "Survey on the novel hybrid aquatic-aerial amphibious aircraft: Aquatic unmanned aerial vehicle (AquaUAV)," Progress in Aerospace Sciences, vol. 74, pp. 131-151, 2014.

[4] M. N. Azzeri, F. A. Adnan, and M. Z. M. Zain, "Review of course keeping control system for unmanned surface vehicle," Jurnal Teknologi, vol. 74, no. 5, pp. 11-20, 2015.

[5] T. I. Fossen and S. P. Berge, "Nonlinear vectorial backstepping design for global exponential tracking of marine vessels in the presence of actuator dynamics," in Proceedings of the IEEE Conference on Decission and Control, pp. 4237-4242, IEEE, San Diego, SD, USA, 1997.

[6] C. Wen, J. Zhou, Z. Liu et al., "Robust adaptive control of uncertain nonlinear systems in the presence of input saturation and external disturbance," System Identification, vol. 56, no. 7, pp. 1672-1678, 2011.

[7] Y. M. Li, S. C. Tong, and T. S. Li, "Direct adaptive fuzzy backstepping control of uncertain nonlinear systems in the presence of input saturation," Neural Computing and Applications, vol. 23, no. 5, pp. 1207-1216, 2013.

[8] M. Chen and W.-H. Chen, "Sliding mode control for a class of uncertain nonlinear system based on disturbance observer," International Journal of Adaptive Control and Signal Processing, vol. 24, no. 1, pp. 51-64, 2010.

[9] A. A. Pereira, J. Binney, G. A. Hollinger, and G. S. Sukhatme, "Risk-aware path planning for autonomous underwater vehicles using predictive ocean models," Journal of Field Robotics, vol. 30, no. 5, pp. 741-762, 2013.

[10] H. Aschemann, "Nonlinear control and disturbance compensation for underactuated ships using extended linearisation techniques," Control Applications in Marine Systems, pp. 167172, 2010.

[11] Y. Yang, J. Du, H. Liu, C. Guo, and A. Abraham, "A trajectory tracking robust controller of surface vessels with disturbance uncertainties," IEEE Transactions on Control Systems Technology, vol. 22, no. 4, pp. 1511-1518, 2013.

[12] D. Swaroop, J. Hedrick K, P. Yip et al., "Dynamic surface control for a class of nonlinear systems," IEEE Transactions on Automatic Control, vol. 45, no. 10, pp. 1893-1899, 2000.

[13] T. I. Fossen and S. I. Sagatun, "Identification of dynamically positioned ships," Modeling Identification \& Control, vol. 17, no. 2, pp. 369-376, 1996. 


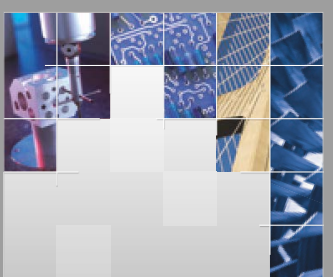

\section{Enfincering}
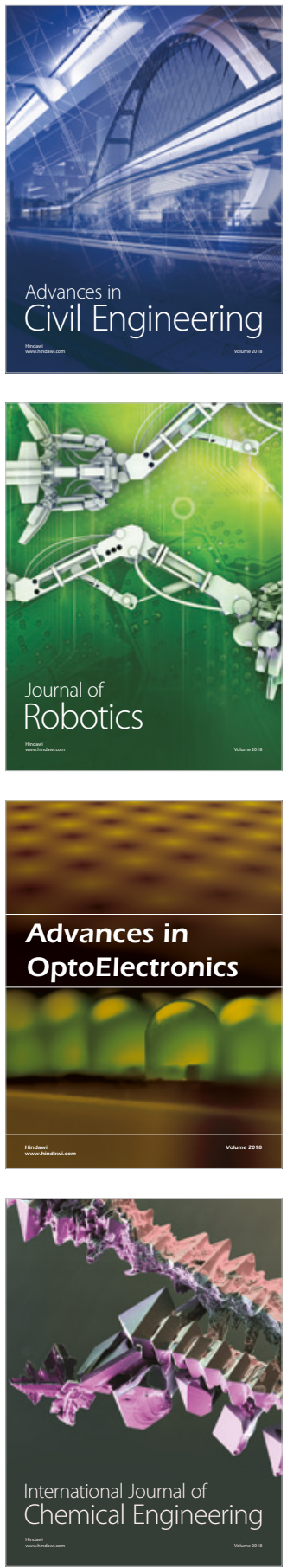

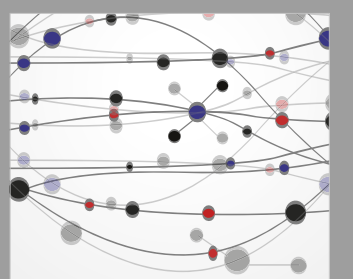

\section{Rotating \\ Machinery}

The Scientific World Journal

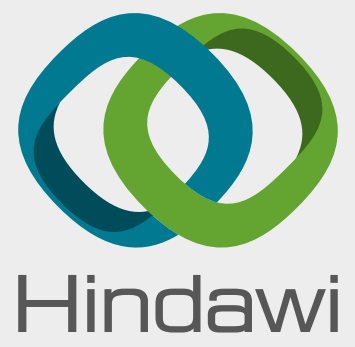

Submit your manuscripts at

www.hindawi.com
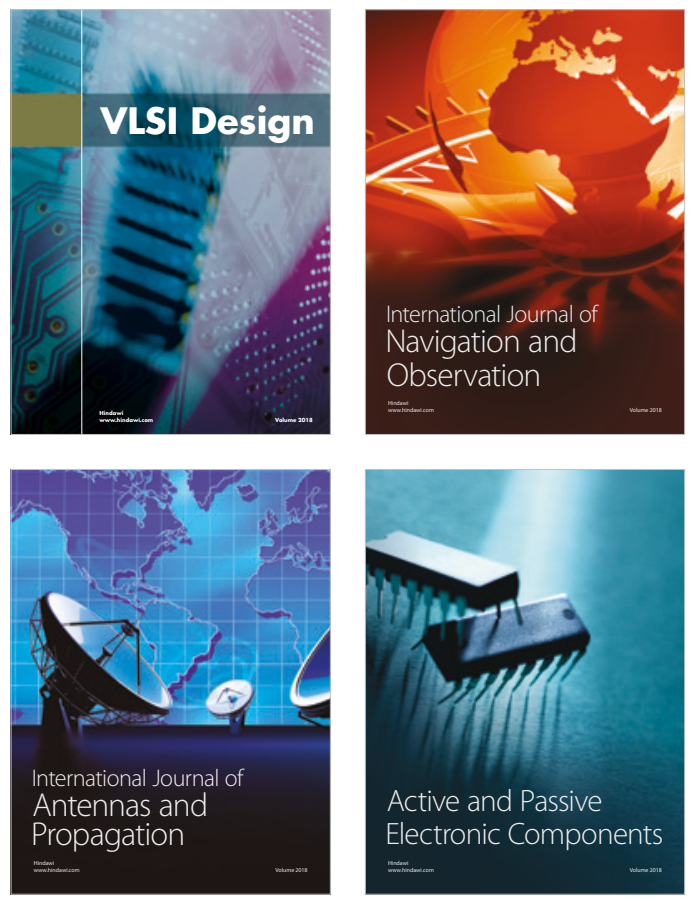
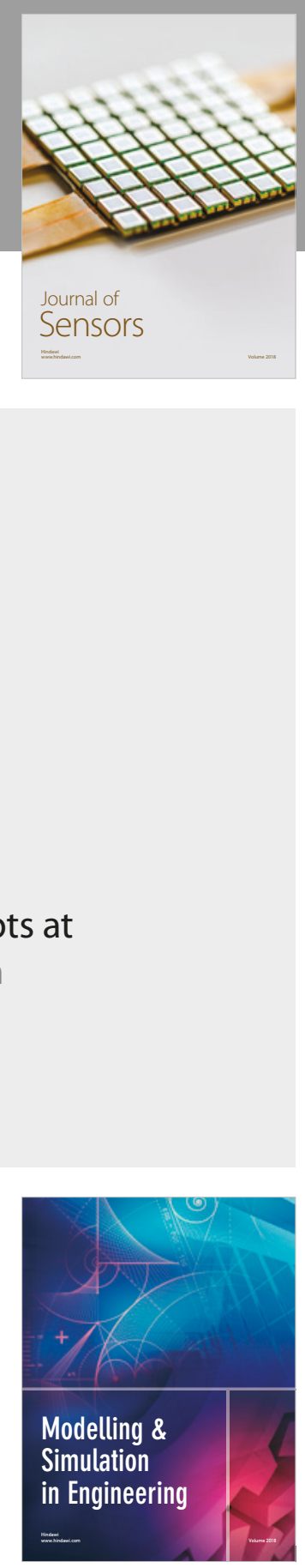

\section{Advances \\ Multimedia}
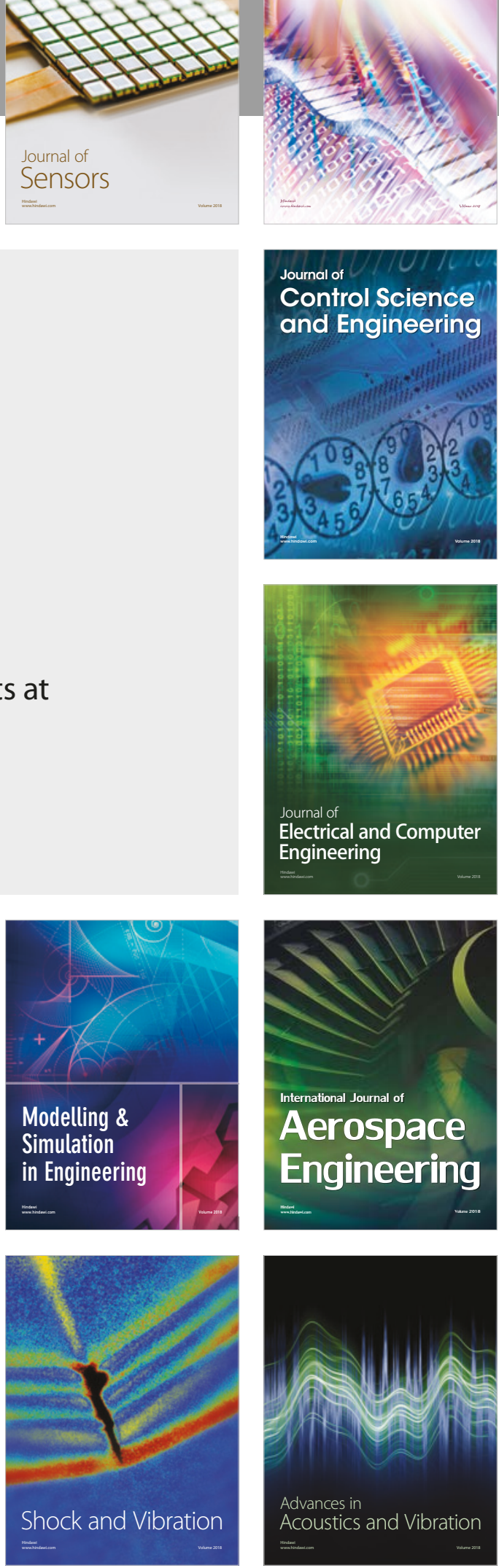